Zhao, L \& Zhang, S. (2013). Development of visual philosophy under impact of philosophy of technology. Journal of Educational Technology Development and Exchange, 6(2), 29-38.

\title{
Development of Visual Philosophy under Impact of Philosophy of Technology
}

\author{
Li Zhao \\ Nanjing Normal University \\ Shuyu Zhang \\ Nanjing Normal University
}

\begin{abstract}
Throughout history the development of technology has been affecting people's lives. Any kind of technological innovation may give a qualitative leap in human society. The impact of technology has penetrated into the study of various disciplines. Philosophy is no exception. In 1877, Karp formally proposed the concept of "philosophy of technology." Technology has been studied from the practical aspect of a tool to the level of philosophy. Although the concept of visual philosophy has not yet been formally proposed, the thinking of philosophical vision has already existed. This paper is to analyze the impact of philosophy of technology on the development of visual philosophy, from the perspective of instrumental theory and humanistic theory, and to clarify current values and directions of visual philosophy.
\end{abstract}

Keywords: development, philosophy of technology, technology, impact, visual philosophy

\section{Introduction}

Philosophy focuses on the origin and essence of objects and concepts, and is the basis and foundation of other superficial research. If someone's study wants to make progress, it must go back to philosophy itself. The study on vision, visual, visual literacy, and visual culture also need to return to the "visual philosophy." There are a lot of achievements on the study of philosophy of technology. The most significant achievement is that people have been able to talk about technical rationality, not just think of it as a tool. In different historical periods of visual philosophy, technology plays an important decisive role. Technology even promotes the development of visual philosophy. Either in the long history of visual philosophy or in today's image world, technology is intertwined with vision. This paper uses the historical and comparative methods to propose the history of visual philosophy, and to examine the influence and value of philosophy of technology on visual philosophy and propose the contemporary research direction and content of visual philosophy.

\section{Philosophy, Philosophy of Technology, Visual Philosophy}

Philosophy is an important tool for the human world to know and transform the world. The object of philosophical research is 
the relationship between human and nature. It gets to the bottom of the problem for an answer. Philosophy of technology and visual philosophy is about specific research in the field of philosophy. Philosophy of technology already has mature system of research. At present, technology has penetrated into every field and subject such as education, psychology, medical science, and also influenced visual philosophy. Although visual philosophy has not been lodged from the history of visual research, it has formed a clear research context. To establish visual philosophy research framework, theories and methods of philosophy and philosophy of technology are helpful for the research of visual philosophy.

\subsection{Philosophy and its Universal Values}

The word philosophy derives from Greek philosophia, formed by Philos and Sophia. Its meaning is "love and wisdom." Philosophy pursues the world's origin, nature, common, or absolute. Its research content is the scientific methodology of understanding and transforming the world. There has always been controversy for "what philosophy is." The study of philosophy lasts a long time. This research field evolving with the development of time has distinct characteristics. Philosophy has produced different branches of philosophy such as natural philosophy and philosophy of science, philosophy of technology, etc. The consensus view of philosophy research is that philosophy is a kind of method. Problems that cannot find the answer can be attributed to the study of philosophy. Philosophy also solves the problems of what objects and effects are like. Meanwhile it can give objects and effects definite descriptions in terms of definitions and concepts. Research on philosophy is based on rational thinking. It is not a simple description of phenomenon, but is based on the nature of thinking. Objects for philosophical research are the universal laws of nature, society, and human minds. Though the description of the philosophy is not the same, from the descriptions above all, one can find the value and significance of the research of philosophy. The reason why philosophy can have a far-reaching effect on other research fields and disciplines is that the philosophy has characteristics of holistic, fundamental, and common sense thinking. Therefore, philosophy has universal significance and value for further study of other fields and disciplines with the long-term development.

\subsection{Philosophy of Technology: Guidance of Philosophy to Technology and Technical Contribution to Philosophy}

The development of technology has been the center of attention for a long time. Technology has been considered to be an important tool for humans to understand and transform the world. The recognition and understanding of technology is stuck on the level of tools and media. So the advance of technology usually refers to the improved tools or media. Technology as tools or media can help humans adapt to the nature. It is the "positive value" of humanity. However, as the intermediary of man and nature, the value of technology and technology alienation bring us to think the value of technology for people. Technology cannot be defined only from the viewpoint of the tool or medium. Technology alienation has brought about the negative effects on humans. Alienation refers to "the subject is in the process of development. Because of its movement the subject produces its opposites (object). This object has become an external alien force against the subject" (Guo, 2002, p. 1). The earliest use of alienation in the research category of philosophy is the German philosopher Hegel. He proposed that alienation refers to "man's creation turns against people and enslaves 
people, and became the alien force to control human" (Wu, 2011, p. 38). From the natural level, technology alienation caused the pollution of the environment, resource crisis, and population expansion; from the social level, technology alienation brings the threat of military technology such as nuclear war; from an ethical level, technology alienation led to chaos of ethical judgment such as the effect of cloning technology on human life. The reason for these phenomena is the nature of technology and the value of technology is like. Whether technical rationality means the technology itself has the ability of rational thinking, or the creation of technology has the ability of reasoning and thinking, the answers to these questions must be searched from philosophy. Philosophy is thinking about the nature of objects and concepts. Any essential research should be attributed to the study of philosophy. Philosophy of technology proposes precisely to solve these problems. In the research process of technology, the generation and development of philosophy of technology needs the enlightenment and guidance of philosophy from the ontological and methodological level.

Secondly, the study of philosophy of technology has made its contribution to the study of philosophy. Since the industrial revolution, the influence of technology has penetrated into various fields and disciplines. Technology has developed from a radical improvement in the level of people's material needs and meeting people's needs for survival. This has challenged people's rational thinking. Philosophy aims to study the relationship between man and nature, and technology plays an important role in the study of the relationship of man and nature. From the perspective of the significance of the relationship of man and nature, philosophy is divided into three branches (Chen, 2012): (1) the narrow natural philosophy, natural ontology; (2) philosophy of science, natural epistemology; and (3) philosophy of technology, natural transformation theory, which shows that research of philosophy of technology plays an important role in the study of philosophy. The emergence of philosophy of technology in 1877 is a direct manifestation of the important impact on the study of philosophy; it is also an important contribution to the study of philosophy. The study of technology shifts also exerts a profound impact on other areas of philosophy, including visual philosophy. This relationship makes philosophy of technology occupy a very important position in the whole philosophy of research areas. The penetration of technical philosophy in other areas is the important value performance in the study of the relationship between man and nature. Fundamentally speaking, the study of philosophy of technology has opened a window to analyze and solve problems from a technical level, and has improved the system of philosophy.

\subsection{The Inevitable Emergence of Visual Philosophy}

Philosophy is a universal sense of ontological and methodological research. The theory and practice of the visual theory also needs to seek the guidance of methodology from philosophy. In the analysis of vision, which serves as a sensory awareness and understanding of a kind of thinking, the interpretation of what the vision is seems not easy. Then, the questions to the principle of vision, visual law, and the relationship of vision and thinking will inevitably go to philosophy to locate the answer. Visual philosophy is philosophy about visual research. This study has existed in the practical activities of the people in the process of understanding and transforming the world. The philosophers, in the ways of thinking relationship between 
man and nature, are impossible to ignore the cognitive effect of the eyes or "window of the soul." Vision and thinking have always been inextricably linked. "More than $70 \%$ of the feeling of receiving of the human body is concentrated on the eyes"(Leicester, 2003, p. 18). To understand the problem of vision is to understand people's thinking. Turning over the history of the study of philosophy, the study of vision philosophical inquiry can be found everywhere. From great thinkers such as Plato and Aristotle, who are builders of visual centrism, to Cassirer, Benjamin, and Heidegger, whose rational understanding and reflection are famous to all. As can be seen, the study of vision does not stay on the superficial surface of visual centrism. In different historical periods, it demonstrates the rich connotation and research to help humans better understand and transform the nature. Visual philosophy has been in progress and it also responds well to related concepts such as "visual centrism." However, visual philosophy just has not yet established a clear and completely theoretical system. Philosophical research methods will guide all kinds of visual research; visual theory and practice research also serve as a contribution to the study of philosophy.

\section{Origins of Visual Philosophy}

From various sources, studies on the philosophical basis of visual culture and the philosophical study on the image culture is not uncommon. But, it is quite rare to clearly clarify the term "visual philosophy," which is not similar to the study of natural philosophy, philosophy of science, philosophy of technology. However, whether in the course of the division of the essence of vision, or a reflection of the current visual appearance and essence, the study on philosophy of "visual" essence has never stopped and will always be extended. Countless sages in history have been discussing this topic such as Plato, Aristotle, Michelle, and so on. From their discussions, two visual clues can be clarified in the philosophical development: the discussion about the "visual centrism" and the shift of philosophy from language to vision.

\subsection{Prosperity and Collapse of Visual Centrism}

Of all the problems, the core of visual philosophy is nature of vision. What is the vision? In the tradition of the western culture, the vision has always been considered to be the noblest of all the senses. Like the soul, vision is like a noble reason existing out of the flesh. The establishment and development of visual centrism can be traced back to ancient Greece, Plato, in the name of Timai Oswald said: "In my opinion, vision is the source of our most useful things ...... God invented vision, and ultimately it is given to us so that we can see the rational process in heaven, and can in turn apply this process to our own rational process." (Lacan, 2005, p. 4). Aristotle, the founder of the visual centrism said in metaphysical, "the pursuit of knowledge is human nature. We are pleased to feel is a description; Even if there is no practical purpose, people love feeling, and among all feelings, vision matters most"(Aristotelian, 1959, p. 1). The status of the visual centrism to ensure the purity of people's philosophical and rational thinking is critical. Visual centrism in modern times still has a lot of supporters. American phenomenologist Hans Jonas, said in a paper entitled noble vision, "only the vision can provide foundation for feeling, through vision the mind can produce long-lasting concept, or the eternal and the ever-present concept", "and thus where vision touches, the mind will be able to reach" (Jonas, 1954, p. 519).

However, with the arrival of the image of the world, structuralism, and post-modern 
thoughts constantly impact on the status of the visual centrism. The visual rational cognitive has been questioned. Omnipresent seeing and be seen are intertwined with confusing eyes. The link between the visible things and its represented invisible aspects is not so tight. What one has seen is not the nature of things. Behind the visual representation there is nothing. Mechanical reproduction in the machine industrial age has created numerous visual impressions, which is dreamy. When the vision sees purely visual representation, there has not been the difference between real and representation, the essence, and phenomenon. From Iraq Debord's "spectacle society" description to Anne Frye Berg interpretation of "the social image valueadded" to Baudrillard's "Simulacra," the attention to visual object features that visual centrism has advocated for is overthrown, and visual philosophy research should focus on visual objects and the possibility mechanism of looking behavior. Thousands of years of visual centrism buildings collapsed in the process of human understanding and transformation of the world. This understanding is an important breakthrough in the visual study of philosophy. If the study on visual philosophy only focuses on visual objects, the audience will be more confusing and fall. At present the important content of studies on visual philosophy is concerning the relationship of seeing and being seen, the mechanisms of looking behavior from "look" to "see" to "read."

\subsection{Shift of Philosophy of Language to Visual Philosophy}

The process of visual centrism from the establishment to the collapse is an explicit clue of the historical development of visual philosophy. From the shift of understanding and characterization of human knowledge, there is an invisible clue for the development of visual philosophy. The attention on visual philosophy from two different shifts is from the peak to the trough and then rising again, moving forward difficultly. An important trend of the 20th century in Western philosophy is a "linguistic shift," in which this understanding is based on modern philosophers' concerning "ideology." Ancient philosophers are concerned more about the elements themselves, what is the look of its own form of things, or visually visible characteristics (i.e., visual centrism). Modern philosophers thinking of an accurate understanding of the concepts must be described from the "concept," which is expressed through language. The status of the noble visual sensory capacities is in decline. The precise degree of expression by language is directly linked with rationality, wisdom, and logic. To the mid-19th century, however, no prophet can predict the emergence of photography, telephones, movies, and their great impact on the language. The concept of "visual culture" during this period, which was the important theoretical results of visual philosophy, was mentioned here. University of Chicago scholars Michelle put forward "visual culture is the culture separated from the rational form of language, increasingly turning to the image centrism, especially to the movie-centric sensibility morphology. Visual Culture not only marks changes and formation of one kind of culture, but also means a conversion of the paradigm of the human mind" (Zhou, 2002, p. 72). Seeing by language is a big step forward in thinking. Language is the spokesperson of wisdom. In the picture era, the visual image can express ideas that language cannot explain. In front of the vivid images, language is pale. Images can also be "read out" beyond language to express the unity of a variety of connotations. The shift from "seeing" by language to using these images to "read," broke the center position of "language." The visual ration has pushed to the foreground. This shift marks visual philosophy is bound to rise. 
Visual image is prevailing today. The status of vision has been highlighted, but thinking of the nature of vision also is puzzling. Today, we do not need to cheer the success of visual shift, but need to focus on the mutual conversion of language and vision. In the visual image world, can language rationality smoothly shift to visual rationality? Maybe this cannot. Reading the text does not mean the capability of reading the image. The lack of visual literacy is an urgent problem. In the same way, visual philosophy will no longer be easily turned to the philosophy of language. People, who used to read images, gradually became poorer in the ability of reading text and expressing by language. This seems to be helpless, but also it extends the research content for visual literacy. The training of visual literacy should be the awareness, understanding, and use of all forms of visual characterization.

\section{The Influence of Philosophy of Technology on Visual Philosophy}

The development of technology which has always focused on the function of technology can be roughly summarized as technical tools theory, technology media theory, technical rationality, and technological humanistic theory. Technology as an important symbol of human progress has been the main tool of the human conquest of nature. It is the grounds of technical tools theory. From ancient times people seek a more high-end, sophisticated and convenient technology to live better lives, thus the technology can be seen as a medium of communication between man and nature. In the face of negative effects brought about by the technology, people have deep thinking about the technology. It is worth studying that technical rationality stems from human or technology itself. Philosophy of technology that aims to find the nature of technology has a profound impact on the development of philosophy. Philosophy of technology also influences the development of research on vision and visual philosophy.

\subsection{Study on the Impact of Technology to the Development of the Visual Philosophy from the Perspective of Instrumental Theory}

Not difficult to see is that the two clues "Visual Centrism and "Visual shift" are in parallel with the development history of visual philosophy. The development of visual philosophy has gone through three historical periods: the era of visual centrism, the era of anti-visual centrism, and visual literacy training period. There are three different tools appearing in the three different periods from the mirror to the camera to the computer. In fact, learning from symbol philosophy proposed by German philosopher Ernst Cassirer, one can find the important revelation of philosophy of technology to visual philosophy implicit in the representation of technical morphological changes.

German philosopher Ernst Cassirer has deep thinking and research on "Symbol Philosophy." He believes that in the course of human evolution, the symbol has also experienced a different evolution. The symbol showed as an icon experiences in the initial stage. Icon is the equivalents of nature, directly facing the nature, and reflects real natural attributes. In this case, the symbol functions like a mirror. The first symbolic development stage corresponds to the period of "mirror" morphology of the technology. A mirror faces the characterization of the object as a technical tool. A mirror reflects a true representation of objects. The visual object and characterization of objects is a peer-to-peer relationship. The elegance and authority of vision established the centrality of the vision.

In the second symbol development historical period, the relationship between the reality and vision had changed. The trait 
between the symbol and the reality was no longer a simple replication relationship, but a causal relationship. There was the complexity of the intermediation process. In this case, the function of a symbol was like a camera. Camera technology principle determines its performance, which no longer simply copies characterized objects. Not only does the lens of a camera have selectivity, but also has a shielding property. Photos only render the tip of the iceberg. The reason why the photo presented to the audience is such a tip of the iceberg, rather than any other part, is the complex causal relationships. Presented characterization does not truly reflect the visual object itself. Getting essential cognition of objects from phenomenon has more obstacles. Looking does not mean seeing, let alone understanding. Pictures of wanton stitching and the use of a variety of technical performance practice, makes the lens show visual objects that are handled and processed artifacts, not natural objects. With the human visual experience and misunderstanding, the magical image confuses eyes. Visual centrism begins to be questioned.

The symbol of its true meaning is in the third period of symbolic development. In this period, symbol and nature is directly separate. If a mirror is the performance of the symbols of nature rendered directly and camera performance is a symbol of natural indirect presentation, "symbol" in this period is not naturally a representation, directly or indirectly, but entirely separate from the raw state of nature and shows as a presentation of the spirit and thinking. The symbols function like computer. Computer as a technology tool, during this period, is the symbol representatives. The abstract of all the symbolic representation is " 0 " and " 1 ," only two figures. Data programming techniques, a simple complex combination of the two figures can refer almost all generations of thinking and command, and has greatly enhanced the speed of people's understanding of transformation of nature. Today, in the image world, images not only affect the process of thinking, but they are the thinking itself. From a phenomenological point of view, something behind the image represented by computer technology tools is completely shelved. Technological image completely changes the original mechanism of visual communication, and these images are entirely under the control of political rights and media. It has become a consensus that visual literacy training is necessary and urgent during this period. Needed is to peel off the external power and politics and face reality and nature. These will be important content of studies on visual philosophy in this period.

\subsection{Examining the Impact of Philosophy of Technology to the Development of the Visual Philosophy from the Perspective of Technology Humanistic Theory}

The understanding of technology as tools is an important factor, which causes the historic absence of philosophy of technology. Although the technology serves as tools to help people understand and transform the world, the understanding of technology has not reached the height of philosophy. Until 1877, the German philosopher Ernst Carp formally proposed the concept of philosophy of technology in his book named Philosophy of Technology Platform. In the mid-18th century, the British Watt's improving the steam engine seemed as the starting point of the first industrial revolution. A series of technological revolutions brought about the shift from manual labor to the production by powered machines. This technology wave was then transmitted to the entire European continent from the United Kingdom, and spread to North America in the 19th century. Technology, which has brought revolutionary change to human society, makes people shift in attitude toward the technology: from disregard to face 
up to the reflection of technology humanistic theory. Technology humanistic theory believes the philosophical thinking about the relationship of human and technology, or the value of technology brought to people. In the early stage technology is created and took advantage of by humans, but as technology advances it has a great deal of positive value. However, when today's technology lead to ecological and natural crisis, people began to reflect on the negative value of the technology.

In the development process of visual philosophy, technology is experiencing from indifference to being confronted with squarely, and then reflecting on the process. In infancy, technology had once dubbed the "witchcraft" title. This slightly derogatory description reflected people's indifferent attitude towards the technology. In this case, the vision was a noble symbol of the thinking. The value of technology to human beings was only one side to reflect reality nature, just like "mirror." It did not predict that technologies would influence the status of vision. Entering the industrial age, people began to face the great strength of technology, especially when technology as a tool to subverted the form and means of visual representation. The technological image makes a mirrorlike vision seem superficial, and the image technology makes vision begin to think. Technology has led to challenges to the visual experience. The visual think is often influenced by technology. People cannot discriminate the boundaries of the essence and phenomenon. At this time of the power of technology, it aims to improve the living conditions of the people and also change the "horizon." Today, technology has brought an even greater crisis to the human's visual experience. The robot can replace humans to complete the specific tasks that human beings cannot complete. Are robots people? Who dare to say that the robots are "Persons" or "Non-persons." Human beings as the master of the world have alternatives. It seems that the whole world can be digitized.

Technology was originally created by people as tools to understand and transform the nature. Today, technology sometimes cannot be in accordance with the established and artificial route that set to transform nature by people, and there has been phenomenon of a breach of human consciousness; the damage to human survival environment and technology alienation. However, the reasons of technology alienation are rooted in the improper values and behavior of human beings who creates and utilizes technology. Based on understanding, the aim of visual literacy education is to enhance literacy training on the aspects of the creation and production of technology. However, the visual representation of the image produced by any technical means, its creators, must be human. Human's rationality has created technical rationality. The correct understanding of the visual audience also depends on the correct understanding of generation mechanism and characterization. It is important enlightenment on visual philosophy that examining the relationship among "people - visual technology" and exploring the value of visual technology to humans on the perspective of the humanistic theory of technology.

\subsection{Values of Philosophy of Technology to the Development of Visual Philosophy}

The study of philosophy of technology not only focuses on the technology itself, but also on the reasonable judgment of the value of technology to humans. The reflection of visual philosophy is not limited to vision. Technological inventions often seem to make the eyes betray the mind. Visual philosophy needs to solve the problems of human beings with technology and technical rationality. The meaning and value of contemporary study of visual philosophy is embodied at this point. 
The content of visual philosophy, in addition to the exploration of the relationship of the eyes, brain, and thinking, also needs to reflect the relationship of human, vision, and technology. This can develop visual literacy education for the audience and the inventor of visual representations. Contemporary theoretical value of visual philosophy is to reconstruct a theory building of visual philosophy through historic and comparison methods, and to make up for the deficiency of the overall architecture of the visual philosophy studies. As a start, we can sort out the history of visual philosophy studies, from vertical perspective, and clarify the characteristics, the main viewpoints and their practice process of each period of development of the visual philosophy. Though the system of visual philosophy has not yet been established, throughout the history of vision research, many research has dubbed the "visual philosophy" name, thus opening up a precedent for the study of visual philosophy such as Germany Nietzsche's perspective "writing instruments which contribute to the formation of ideas," Cassirer's theory symbol philosophy, Rudolf Arnheim's "visual thinking," and Walter Benjamin concentrating on the technology image and its rich achievements of the revolution triggered in the visual field (Meng, 2005, 4-10). We can stand on the shoulders of these sages to see farther and higher. Then, comparison methods can help us understand the relationship of visual philosophy and its related fields or subjects. In the foundation of rich research results of humanism and phenomenology, construct theoretical system of visual philosophy.

The practical value of studies on visual philosophy is to solve the real problem of how to interpret the image of the world. Current theoretical assumptions and studies of the vision focus on the understanding of the visual mechanism or the way of visual representation, and strategies of visual literacy training. These studies examine visual practice, but its goal is more realistic to better cope with the visual research. However in the final analysis, this is an assumption from the theoretical level. Such proposed research is a "stopgap measure" that does not solve the rooted cause of the problems in the practical study of visual philosophy. Digitization and virtualization are two key words in today's image era of visual representation. The practical research of visual philosophy should focus on the characteristic features of era and technical rational thinking to make full use of technology to create a better and more beautiful visual representation (i.e., visual educational resources) to carry out the actual social action and specific projects. Finally, people will realize the concept and strategies of visual literacy education through their participation in the process of practice. Since birth, the world's first touch is the "light of life" through vision. Visual perception accompanies a person's whole life. It is important that we have been concerned about the "look" ability and visual experience. Therefore, the understanding of the vision, since ancient times, is an eternal topic. Research on establishment and development of visual philosophy applies to daily activities. It needs to restructure ways of history and comparison. Due to the impact of technology and philosophy of technology, the development of the visual philosophy is endowed with new meanings and value. In terms of the "Then Time," "Current Time," and "Future Time," the power of technology will continue to affect people's practical activities of understanding and transforming the nature through vision.

\section{References}

Aristotelian. (1959). Metaphysics. Beijing, China: Commercial Press.

Chen Chang shu. (2012). Discuss on Philosophy of technology . Beijing, China: Science Press. 
Lacan, J. (2005). the Wonders of visual culture: Visual Culture General. Beijing, China: China Renmin University Press.

Meng Jian. (2005) Image era: the visual culture propagation theory interpretation. Shanghai: Fudan University Press.

Leicester, P. M. (2003). Visual communication image contains dynamic information. Beijing, China: Beijing Broadcasting Institute Press.

Zhang Zhiwei. (2012). Western philosophy fifteen stresses. Beijing, China: Peking University Press.

Zhou Xian. (2002). Interpret blueprints body ideology. Tianjin, China: Tianjin Academy of Social Sciences Press.

Guo Chongchen, \& Chen Fan.(2002). View on Value of Technology alienation. Science technology and Dialectics. 19(1),1-5.

Hans Jonas. (1954). The Nobility of Sight. Philosophy And Phenomenological Research, 14(4), 519-523.

Wu Junjie, \&Zhang Xinming. (2011). Implications of technological alienation theory on multimedia teaching. Primary and Middle School Educational Technology, (12 ), 38-40.

\section{Acknowledgments}

This work is funded by the Priority Academic Program Development of Jiangsu Higher Education Institution, and Humanities and Social Sciences Projects of Ministry of education of China under Grant No. 12YJA880161.

\section{Contact the Authors}

\section{Li Zhao}

Nanjing Normal University

E-mail:20190231@qq.com

\section{Shuyu Zhang}

Nanjing Normal University

E-mail:glasgowzhang123@163.com 